CERN-TH.7358/94

BARI-TH/181-94

\title{
Standard solar model uncertainties and their correlations in the analysis of the solar neutrino problem
}

\author{
G.L. Fogli ${ }^{(b)}$ and E. Lisi ${ }^{(a, b)}$ \\ (a) Theory Division, CERN, Geneva, Switzerland \\ (b) Dipartimento di Fisica di Bari, Bari, Italy \\ Sezione INFN di Bari, Bari, Italy
}

\begin{abstract}
We present here an analysis of the theoretical uncertainties associated to the predicted solar neutrino rates for the current gallium, chlorine and water-Cherenkov experiments. We estimate the theoretical rates and their error matrices in three independent solar models (proposed by Bahcall and Pinsonneault, Turck-Chièze and Lopes, and Dar and Shaviv), and compare them to the experimental data. We show that the correlations of the theoretical uncertainties are crucial to assess the statistical significance of the solar neutrino deficit. The implications of a parametrization of the errors in terms of the central temperature of the Sun are also discussed.
\end{abstract}

CERN-TH.7358/94

BARI-TH/181-94

July 1994 


\section{Introduction}

One of the most challenging problems in the field of astroparticle physics is the long-standing discrepancy between measured and predicted solar neutrino rates. The most recent results of the GALLEX [1] and SAGE [2] gallium (Ga) experiments, Homestake chlorine $(\mathrm{Cl})$ experiment [3], and Kamiokande (Kam) waterCherenkov experiment [4] are collected in Table 1. The measured solar neutrino fluxes appear to be systematically lower than theoretically predicted in a variety of standard solar models (SSMs), in particular in the detailed models proposed by Bahcall and Ulrich [5], Bahcall and Pinsonneault [6] and Turck-Chièze and Lopes [7]. The deficit seems to be substantially reduced in the SSM recently proposed by Dar and Shaviv [8]. For the sake of brevity, in the following we will refer to the previous four models as BU, BP, TL and DS respectively.

The critical comparison of the different assumptions underlying the various SSMs is the preferred subject of discussion among model builders, and it will not be addressed in this paper. We prefer instead to investigate accurately the compatibility of the different theoretical predictions with the experimental data. To this aim, in Sec. 2 we consider in detail the analytical prescription required to estimate the theoretical error matrix. In particular, we provide all the tools for a do-it-yourself estimate of the errors. In Sec. 3 we apply this general method to three independent solar models (BP, TL and DS), in order to assess the statistical significance of the neutrino deficit. The implications of charting the different solar models according to their predicted core temperature $T_{C}$ are discussed in Sec. 4. In Sec. 5 we finally summarize our conclusions about the present status of the solar neutrino problem.

Table 1: Measured neutrino rates and $1 \sigma$ total experimental errors for the gallium $(\mathrm{Ga})$, chlorine $(\mathrm{Cl})$ and water-Cherenkov $(\mathrm{Kam})$ experiments. The Kam rate is conventionally normalized to the BU standard solar model predictions (BUSSM).

\begin{tabular}{lccccc}
\hline \hline \multicolumn{1}{c}{ Experiment } & Ref. & Rate & Error & Units \\
\hline GALLEX+SAGE & $(\mathrm{Ga})$ & {$[1,2]$} & 78 & 10 & SNU \\
Homestake & $(\mathrm{Cl})$ & {$[3]$} & 2.55 & 0.25 & SNU \\
Kamiokande & $(\mathrm{Kam})$ & {$[4]$} & 0.50 & 0.07 & Kam/BUSSM \\
\hline \hline
\end{tabular}




\section{Analytical estimate of the theoretical errors}

In any solar model, the predicted neutrino rates $R_{j}$ (in the following $j=1,2,3$ stands for $\mathrm{Ga}, \mathrm{Cl}, \mathrm{Kam}$ ) are affected by - in general correlated - theoretical uncertainties. In this section we describe in detail a prescription useful to estimate the full theoretical error matrix analytically ${ }^{1}$. As we will see in the following, Tables from 2 to 6 collect all needed input data.

In Table 2 we report the eight relevant neutrino fluxes $\left\{\phi_{i}\right\}_{i=1, \ldots, 8}$ (pp, pep, hep, Be, B, N, O, F) as estimated in the BU, BP, TL and DS solar models.

The fluxes in Table 2, when multiplied by the energy-averaged detector crosssection factors $\left\{C_{i j}\right\}_{j=1, \ldots, 3}^{i=1, \ldots, 8}$ reported in Table 3, give the theoretical partial rates $R_{i j}\left(R_{i j}=C_{i j} \phi_{i}\right)$ and the total rates $R_{j}\left(R_{j}=\sum_{i=1}^{8} R_{i j}\right)$ for the three different types of solar neutrino experiments.

Table 2: Neutrino fluxes $\phi_{i}\left(\mathrm{~cm}^{-2} \mathrm{~s}^{-1}\right)$ in the BU, BP, TL and DS models.

\begin{tabular}{|c|c|c|c|c|c|c|c|c|}
\hline $\begin{array}{c}\text { Reference } \\
\text { solar } \\
\text { model }\end{array}$ & $\begin{array}{l}\underset{\times}{\mathrm{pp}} \\
10^{10}\end{array}$ & $\begin{array}{l}\text { pep } \\
\times \\
10^{8}\end{array}$ & $\begin{array}{c}\text { hep } \\
\times \\
10^{3}\end{array}$ & $\begin{array}{c}\mathrm{Be} \\
\times \\
10^{9}\end{array}$ & $\begin{array}{c}\mathrm{B} \\
\times \\
10^{6}\end{array}$ & $\begin{array}{c}\mathrm{N} \\
\times \\
10^{8}\end{array}$ & $\begin{array}{c}\mathrm{O} \\
\times \\
10^{8}\end{array}$ & $\begin{array}{c}\mathrm{F} \\
\times \\
10^{6}\end{array}$ \\
\hline $\mathrm{BU} \quad[5]$ & 6.00 & 1.40 & 7.58 & 4.69 & 5.76 & 6.09 & 5.22 & 5.16 \\
\hline $\mathrm{BP} \quad[6]$ & 6.00 & 1.43 & 1.23 & 4.89 & 5.69 & 4.92 & 4.26 & 5.39 \\
\hline $\mathrm{TL} \quad[7]$ & 6.03 & 1.39 & - & 4.34 & 4.43 & 3.83 & 3.18 & - \\
\hline $\mathrm{DS} \quad[8]$ & 6.04 & 1.40 & - & 4.30 & 2.77 & 0.747 & 0.217 & 5.21 \\
\hline
\end{tabular}

Table 3: Energy-averaged detection cross sections $C_{i j}$, taken from the BU paper [5]. The same matrix is used by TL. The elements $\left(C_{32}, C_{52}\right)$ are updated in $\mathrm{BP}$ from the old $\mathrm{BU}$ values $(3.90,1.06)$ to the new values $(4.26,1.09)$. Units are such that the expected rates $C_{i j} \phi_{i}$ are directly given in $\mathrm{SNU}$ for the $\mathrm{Ga}$ and $\mathrm{Cl}$ experiments, and as a ratio to the BU predicted rate for Kamiokande (Kam/BUSSM).

\begin{tabular}{|c|c|c|c|c|c|c|c|c|}
\hline Experiment & $\begin{array}{c}\underset{\times}{\operatorname{pp}} \\
10^{-9}\end{array}$ & $\begin{array}{c}\text { pep } \\
\times \\
10^{-8}\end{array}$ & $\begin{array}{c}\text { hep } \\
\times \\
10^{-6}\end{array}$ & $\begin{array}{c}\mathrm{Be} \\
\stackrel{\times}{10^{-9}}\end{array}$ & $\stackrel{\stackrel{B}{\times}}{10^{-6}}$ & $\begin{array}{c}\stackrel{N}{\times} \\
10^{-9}\end{array}$ & $\begin{array}{c}\stackrel{\mathrm{O}}{\times} \\
10^{-8}\end{array}$ & $\underset{\stackrel{\mathrm{F}}{\times}}{10^{-8}}$ \\
\hline $\mathrm{Ga}$ & 1.18 & 2.15 & 7.30 & 7.32 & 2.43 & 6.18 & 1.16 & 1.17 \\
\hline $\mathrm{Cl}$ & 0 & 0.16 & 3.90 & 4.89 & 1.06 & 0.17 & 0.07 & 0.07 \\
\hline Kam & 0 & 0 & 0.66 & 0 & 0.173 & 0 & 0 & 0 \\
\hline
\end{tabular}

\footnotetext{
${ }^{1}$ We used this method in previous works [9, 10]; an explicit presentation of it (not as complete as here) has been given in Ref. [11].
} 
The accuracy in the theoretical estimate of the three neutrino rates $R_{j}$ is limited by:

1) The uncertainties in the capture cross-section of each detector. These are completely specified by the uncorrelated relative errors $\Delta \ln C_{i j}$ affecting the cross-section factors $C_{i j}$, as given in Table 4 .

2) The uncertainties in the astrophysical parameters used as input in building the different solar models. Their relevant components can be traced back to the nuclear $S$-factors $S_{11}, S_{33}, S_{34}, S_{1,14}, S_{17}$, the metallicity $(Z / X)$, the Sun age $(A g e)$ and the opacity $(O p a)$. We denote these input parameters ${ }^{2}$ as $\left\{X_{k}\right\}_{k=1, \ldots, 9}$. The corresponding relative errors $\Delta \ln X_{k}$, as adopted by $\mathrm{BU}, \mathrm{BP}, \mathrm{TL}$ and DS, are reported in Table 5. Notice that the estimate of $\Delta \ln X_{k}$ is more conservative in TL than in BU and BP. We will assume the TL values for the missing entries in the fourth row of Table 5 (DS model).

Table 4: $1 \sigma$ relative errors $\Delta \ln C_{i j}$ of the energy-averaged cross-sections, taken from the $\mathrm{BU}, \mathrm{BP}$ papers.

\begin{tabular}{ccccccccc}
\hline \hline Experiment & $\mathrm{pp}$ & pep & hep & $\mathrm{Be}$ & $\mathrm{B}$ & $\mathrm{N}$ & $\mathrm{O}$ & $\mathrm{F}$ \\
\hline $\mathrm{Ga}$ & 0.025 & 0.070 & 0.310 & 0.033 & 0.300 & 0.035 & 0.065 & 0.065 \\
$\mathrm{Cl}$ & 0 & 0.020 & 0.033 & 0.020 & 0.033 & 0.020 & 0.020 & 0.020 \\
$\mathrm{Kam}^{*}$ & 0 & 0 & 0 & 0 & 0 & 0 & 0 & 0 \\
\hline \hline
\end{tabular}

${ }^{*}$ Radiative correction uncertainties of the scattering cross section are negligible.

Table 5: $1 \sigma$ relative errors $\Delta \ln X_{k}$ of the nine relevant input parameters $X_{k}$, adopted in the BU, BP, TL and DS solar models.

\begin{tabular}{cccccccccc}
\hline \hline SSM & $S_{11}$ & $S_{33}$ & $S_{34}$ & $S_{1,14}$ & $S_{17}$ & Lum & $Z / X$ & Age & Opa \\
\hline BU & 0.017 & 0.057 & 0.020 & 0.120 & 0.073 & 0.002 & 0.063 & 0.007 & 0.010 \\
BP & 0.015 & 0.060 & 0.032 & 0.120 & 0.090 & 0.002 & 0.063 & 0.007 & 0.020 \\
TL & 0.040 & 0.050 & 0.040 & - & 0.130 & 0.005 & 0.100 & 0.010 & 0.050 \\
DS & - & - & 0.067 & - & 0.120 & - & - & - & - \\
\hline \hline
\end{tabular}

\footnotetext{
${ }^{2}$ Actually, the opacity is itself a whole set of input parameters (opacity tables). However, changes in the opacity tables produce highly correlated shifts in the fluxes $\phi_{i}$, as they were due to a single parameter $(\mathrm{Opa})$.
} 
The error propagation from $X_{k}$ to $\phi_{i}$ is governed by the matrix $\left\{\alpha_{i k}\right\}$ of the $\operatorname{logarithmic}$ derivatives $\alpha_{i k}=\partial \ln \phi_{i} / \partial \ln X_{k}$, whose numerical estimate, as performed by Bahcall and collaborators, is reported in Table 6. The last row is obtained by comparing the output fluxes relative to models with different opacity input tables $[5,6]$. Several entries of this matrix have been independently estimated by the authors of Refs. [7, 12], which quote a good agreement with the $\mathrm{BU}, \mathrm{BP}$ values. The only element of $\left\{\alpha_{i k}\right\}$ quoted in the Kovetz and Shaviv model [13] (on which the DS model is based) is $\alpha_{56}=7.1$, close to the value 6.76 of Table 6. At this point, it seems reasonable to use the same matrix $\left\{\alpha_{i k}\right\}$ as that in Table 6 for all models.

Table 6: Logarithmic derivatives $\alpha_{i k}=\partial \ln \phi_{i} / \partial \ln X_{k}$, taken from [5, 6].

\begin{tabular}{lcccccccc}
\hline \hline$X_{k} \backslash \phi_{i}$ & pp & pep & hep & Be & B & N & O & F \\
\hline$S_{11}$ & +0.14 & -0.17 & -0.08 & -0.97 & -2.59 & -2.53 & -2.93 & -2.94 \\
$S_{33}$ & +0.03 & +0.05 & -0.45 & -0.43 & -0.40 & +0.02 & +0.02 & +0.02 \\
$S_{34}$ & -0.06 & -0.09 & -0.08 & +0.86 & +0.81 & -0.05 & -0.05 & -0.05 \\
$S_{1,14}$ & -0.02 & -0.02 & -0.01 & +0.00 & +0.01 & +0.85 & +1.00 & +0.01 \\
$S_{17}$ & +0.00 & +0.00 & +0.00 & +0.00 & +1.00 & +0.00 & +0.00 & +0.00 \\
$L u m$ & +0.73 & +0.87 & +0.12 & +3.40 & +6.76 & +5.16 & +5.94 & +6.25 \\
$Z / X$ & -0.08 & -0.17 & -0.22 & +0.58 & +1.27 & +1.86 & +2.03 & +2.09 \\
Age & -0.07 & +0.00 & -0.11 & +0.69 & +1.28 & +1.01 & +1.27 & +1.29 \\
Opa & +0.14 & +0.24 & +0.54 & -1.38 & -2.60 & -1.68 & -2.05 & -2.15 \\
\hline \hline
\end{tabular}

The entries of the matrix $\left\{\alpha_{i k}\right\}$ are not linearly independent. In fact, the fluxes are constrained by the equation of energy production (see, e.g., Ref. [14])

$$
\sum_{i=1}^{8}\left(Q-2 \bar{E}_{i}\right) \phi_{i}=2 K
$$

where $Q=26.73 \mathrm{MeV}, \bar{E}_{i}$ is the $i$-th flux average energy (in $\mathrm{MeV}$ ), and $K$ is the solar constant $\left(K=0.849 \times 10^{12} \mathrm{MeV} \mathrm{cm}^{-2} \mathrm{~s}^{-1}\right)$, proportional to the luminosity ( $L u m=X_{6}$ ). By considering the flux variations induced by the $X_{k}$ uncertainties, the above equation leads to the following interesting sum rule

$$
\frac{\sum_{i=1}^{8}\left(Q-2 \bar{E}_{i}\right) \phi_{i} \alpha_{i k}}{\sum_{i=1}^{8}\left(Q-2 \bar{E}_{i}\right) \phi_{i}}=\delta_{k 6} \quad(k=1, \ldots, 9)
$$


which is satisfied at the $2 \%$ level by using BU or BP fluxes. This means that energy conservation is correctly implemented: a perturbation of $X_{k}$ with $k=6$ $(k \neq 6)$ induces a redistribution of the fluxes while varying (keeping constant) $K$, as it should be.

Now we calculate the relevant error matrices. As said before, the matrix $\sigma_{j_{1} j_{2}}^{2}(\mathrm{TH})$ of the total theoretical errors affecting the rates $R_{j}$ is the sum of two distinct matrices, one with the errors due to the detector cross sections (CS errors) and the other constructed with the uncertainties concerning the astrophysical parameters (AP errors) :

$$
\sigma_{j_{1} j_{2}}^{2}(\mathrm{TH})=\sigma_{j_{1} j_{2}}^{2}(\mathrm{CS})+\sigma_{j_{1} j_{2}}^{2}(\mathrm{AP}) \quad\left(j_{1}, j_{2}=1, \ldots, 3\right)
$$

The contributions to $\sigma_{j_{1} j_{2}}^{2}(\mathrm{CS})$, coming from the uncertainties $\Delta \ln C_{i j}$ in the detector cross sections, are uncorrelated in both the indices $(i, j)$, and give rise to the diagonal matrix

$$
\begin{aligned}
\sigma_{j_{1} j_{2}}^{2}(\mathrm{CS}) & =\delta_{j_{1} j_{2}} \sum_{i_{1}=1}^{8} \sum_{i_{2}=1}^{8} \delta_{i_{1} i_{2}} \frac{\partial R_{j_{1}}}{\partial \ln C_{i_{1} j_{1}}} \frac{\partial R_{j_{2}}}{\partial \ln C_{i_{2} j_{2}}} \Delta \ln C_{i_{1} j_{1}} \Delta \ln C_{i_{2} j_{1}} \\
& =\delta_{j_{1} j_{2}} \sum_{i=1}^{8} R_{i j_{1}}^{2}\left(\Delta \ln C_{i j_{1}}\right)^{2} .
\end{aligned}
$$

The contributions to $\sigma_{j_{1} j_{2}}^{2}(\mathrm{AP})$, coming from the uncertainties in the input parameters $X_{k}$, affect all the fluxes $\phi_{i}$ systematically and give rise to off-diagonal elements in the error matrix

$$
\begin{aligned}
\sigma_{j_{1} j_{2}}^{2}(\mathrm{AP}) & =\sum_{k=1}^{9} \frac{\partial R_{j_{1}}}{\partial \ln X_{k}} \frac{\partial R_{j_{2}}}{\partial \ln X_{k}}\left(\Delta \ln X_{k}\right)^{2} \\
& =\sum_{i_{1}=1}^{8} \sum_{i_{2}=1}^{8} R_{i_{1} j_{1}} R_{i_{2} j_{2}} \sum_{k=1}^{9} \alpha_{i_{1} k} \alpha_{i_{2} k}\left(\Delta \ln X_{k}\right)^{2}
\end{aligned}
$$

The sum of the theoretical and experimental error matrices gives the total error matrix: denoting by $\sigma_{j_{1}}^{e x p}$ the experimental errors given in Table 1 ,

$$
\begin{aligned}
\sigma_{j_{1} j_{2}}^{2}(\mathrm{TOT}) & =\sigma_{j_{1} j_{2}}^{2}(\mathrm{TH})+\sigma_{j_{1} j_{2}}^{2}(\mathrm{EXP}) \\
& =\sigma_{j_{1} j_{2}}^{2}(\mathrm{TH})+\delta_{j_{1} j_{2}} \sigma_{j_{1}}^{\exp } \sigma_{j_{2}}^{\exp } .
\end{aligned}
$$


At this point, the statistical significance of the solar neutrino deficit is measured by the global $\chi^{2}$ function

$$
\chi^{2}=\sum_{j_{1}, j_{2}=1}^{3}\left(R_{j_{1}}-R_{j_{1}}^{e x p}\right)\left[\sigma_{j_{1} j_{2}}^{2}(\mathrm{TOT})\right]^{-1}\left(R_{j_{2}}-R_{j_{2}}^{e x p}\right)
$$

Finally, it is useful to define also the $8 \times 8$ flux error matrix $\sigma_{i_{1} i_{2}}^{2}$, which receives only "astrophysical" contributions

$$
\begin{aligned}
\sigma_{i_{1} i_{2}}^{2} & =\sum_{k=1}^{9} \frac{\partial \phi_{i_{1}}}{\partial \ln X_{k}} \frac{\partial \phi_{i_{2}}}{\partial \ln X_{k}}\left(\Delta \ln X_{k}\right)^{2} \\
& =\phi_{i_{1}} \phi_{i_{2}} \sum_{k=1}^{9} \alpha_{i_{1} k} \alpha_{i_{2} k}\left(\Delta \ln X_{k}\right)^{2}
\end{aligned}
$$

The analytical formulae for the error matrices reported above can be compared with some numerical estimates performed in the literature. In particular, values of $\sqrt{\sigma_{i i}}$ and of some of their single astrophysical (AP) components have been published in Table XIII of Ref. [5] and Table VII of Ref. [6]. Values of $\sqrt{\sigma_{j j}(\mathrm{TH})}$ and of some of their astrophysical (AP) and detector cross-section (CS) components are given in Table XVI of Ref. [5], Table X of Ref. [6] and Table VI of Ref. [7]. Our results agree with all these published values with great accuracy when the appropriate input for each reference model is used.

It is very important to test now the off-diagonal elements of the error matrix, generated by the astrophysical (AP) uncertainties. To this aim we use the set of 8-plets of fluxes $\phi_{i}$ obtained by Bahcall in a Monte Carlo evaluation of "1000 standard solar models" ${ }^{3}$. These 1000 different models are generated by randomly varying (according to Gaussian distributions) the five input parameters $S_{11}, S_{33}$, $S_{34}, S_{17}, Z / X\left\{X_{k}, k=1,2,3,5,7\right\}$. If we now restrict the $k$-summation in Eq. (8) to $\{k=1,2,3,5,7\}$ and calculate analytically the flux correlation matrix $\rho_{i_{1} i_{2}}=\sigma_{i_{1} i_{2}}^{2} / \sqrt{\sigma_{i_{1} i_{1}}^{2} \sigma_{i_{2} i_{2}}^{2}}$, we obtain the values reported in the upper half of Table 7 . In the same table (lower half) we report the numerical correlations of the 1000 SSM fluxes. The non-trivial agreement of all the off-diagonal entries completes the desired check of Eqs. (3-6), which will be at the basis of the analysis presented in the next section.

\footnotetext{
${ }^{3}$ We thank J.N. Bahcall for kindly providing us with the results of his 1000 SSM Monte Carlo.
} 
It is interesting to observe that as inputs of our analytical method we require the outputs of $\mathcal{O}(N)$ solar model replicas (for example, to estimate the $\alpha_{i k}$ 's), where $N$ is the number of astrophysical parameters used as variables (whith $N=9$ in our most general case, $N=5$ in Table 7 ). Conversely, a full Monte Carlo evaluation of the errors requires $\mathcal{O}\left(N^{m}\right)$ solar code runs, where $m$ is the number of sampling points in the Gaussian distributions of the $N$ variables: the Monte Carlo method is thus intrinsically limited by computing time if the effects of a large number of parameters are to be studied.

Table 7: Off-diagonal elements of the flux error matrix. Upper half: our estimate of the flux correlation matrix $\rho_{i_{1} i_{2}}$, using $\mathrm{BU}$ input data and taking into account only the errors induced by $S_{11}, S_{33}, S_{34}, S_{17}, Z / X$. Lower half: correlation matrix extracted from the 1000 SSM Monte Carlo simulation of Bahcall.

\begin{tabular}{|c|c|c|c|c|c|c|c|c|}
\hline$\phi_{i_{1}} \backslash \phi_{i_{2}}$ & $\mathrm{pp}$ & pep & hep & $\mathrm{Be}$ & B & $\mathrm{N}$ & $\mathrm{O}$ & $\mathrm{F}$ \\
\hline $\mathrm{pp}$ & 1 & & & & & & & \\
\hline pep & 0.784 & 1 & & & & & & \\
\hline hep & 0.142 & 0.242 & 1 & & & & & \\
\hline $\mathrm{Be}$ & -0.960 & -0.766 & 0.080 & 1 & & & & \\
\hline B & -0.792 & -0.591 & -0.139 & 0.746 & 1 & & & \\
\hline $\mathrm{N}$ & -0.928 & -0.778 & -0.437 & 0.792 & 0.748 & 1 & & \\
\hline $\mathrm{O}$ & -0.930 & -0.767 & -0.432 & 0.793 & 0.751 & 1.000 & 1 & \\
\hline $\mathrm{F}$ & -0.930 & -0.772 & -0.433 & 0.793 & 0.750 & 1.000 & 1.000 & 1 \\
\hline $\mathrm{pp}$ & 1 & & & & & & & \\
\hline pep & 0.770 & 1 & & & & & & \\
\hline hep & 0.077 & 0.284 & 1 & & & & & \\
\hline $\mathrm{Be}$ & -0.916 & -0.693 & 0.046 & 1 & & & & \\
\hline B & -0.727 & -0.503 & -0.157 & 0.742 & 1 & & & \\
\hline $\mathrm{N}$ & -0.885 & -0.735 & -0.461 & 0.796 & 0.729 & 1 & & \\
\hline $\mathrm{O}$ & -0.886 & -0.718 & -0.453 & 0.800 & 0.734 & 1.000 & 1 & \\
\hline $\mathrm{F}$ & -0.886 & -0.721 & -0.454 & 0.800 & 0.734 & 1.000 & 1.000 & 1 \\
\hline$\phi_{i_{1}} / \phi_{i_{2}}$ & $\mathrm{pp}$ & pep & hep & $\mathrm{Be}$ & $\mathrm{B}$ & $\mathrm{N}$ & $\mathrm{O}$ & $\mathrm{F}$ \\
\hline
\end{tabular}




\section{An analysis in three different solar models}

The analytic prescription of the previous section is now applied to the BP, TL and DS solar models. They can be thought of as representative of models yielding "high" (BP), "intermediate" (TL) and "low" (DS) neutrino rates, as a result of different - although "standard" — physical assumptions.

The main results are collected in Table 8, were we report, for each of the three reference models, the predicted rates $R_{j}$, the total theoretical errors $\sqrt{\sigma_{j j}(\mathrm{TH})}$ with their correlations, and the global $\chi^{2}$ (including or not the correlation effects).

Let us stress that the correlations of the theoretical errors in Table 8 are sizeable, and it would be a gross approximation, in principle, to neglect them in the $\chi^{2}$ estimate, as can be seen by comparing the last two columns of the table. The impact of the correlation matrix is often ignored when the statistical significance of the solar neutrino problem is criticized [15]. The "uncorrelated" approximation is particularly dangerous in the DS model, where it leads to a much lower $\chi^{2}$ (13.5 instead of 20.5). The effect is opposite in the BP model, and happens to be negligible in the TL model. We will turn again to this point.

Among the three models, BP has the highest $\chi^{2}$, as a result of higher predicted rates and relatively smaller theoretical input errors. However, even in the DS model (lower rates, conservative errors) the value assumed by the global $\chi_{\text {сотт }}^{2}$ is certainly still large enough to elevate the solar neutrino deficit to the status of a problem.

Table 8: Theoretical rates, with their errors and correlations, and global $\chi^{2}$ [with (corr) and without (uncorr) correlations] in the BP, TL and DS models. Units as in Table 1.

\begin{tabular}{rrrrrcccc}
\hline \hline SSM & EXP & Rate & $1 \sigma$ err. & Correlation matrix & $\chi_{\text {cotr }}^{2}$ & $\chi_{\text {uncort }}^{2}$ \\
\hline \multirow{3}{*}{$\mathrm{BP}$} & $\mathrm{Ga}$ & 131.55 & 6.41 & 1 & & & & \\
& $\mathrm{Cl}$ & 7.98 & 1.00 & 0.628 & 1 & & 44.0 & 58.0 \\
& $\mathrm{Kam}$ & 0.99 & 0.14 & 0.620 & 0.975 & 1 & & \\
\hline \multirow{2}{*}{$\mathrm{TL}$} & $\mathrm{Ga}$ & 122.66 & 7.43 & 1 & & & & \\
& $\mathrm{Cl}$ & 6.24 & 1.34 & 0.820 & 1 & & 23.3 & 21.8 \\
& $\mathrm{Kam}$ & 0.77 & 0.19 & 0.808 & 0.991 & 1 & & \\
$\mathrm{DSS}$ & $\mathrm{Ga}$ & 113.26 & 5.33 & 1 & & & & \\
& $\mathrm{Cl}$ & 4.31 & 0.87 & 0.792 & 1 & & 20.5 & 13.5 \\
\hline \hline
\end{tabular}


We must note at this point that our $\mathrm{Ga}$ and $\mathrm{Cl}$ rates for the DS model in Table 8 are slightly larger than those quoted by the authors (109 and 4.2 SNU respectively, see Ref. [8]). This disturbing difference implies that Dar and Shaviv use an alternative, but unspecified, set of cross-section factors $C_{i j}$. However, even using their $\mathrm{Ga}$ and $\mathrm{Cl}$ quoted rates, we get $\chi_{\text {corr }}^{2}=17.2$, which still implies a very low confidence level.

We now give a graphical representation of the information contained in Tables 1 and 8 , in order to make more evident the "distance" between theory and experiment. In Figs. 1, 2 and 3, using as coordinates the total rates $R_{j}$ $(j=1,2,3=\mathrm{Ga}, \mathrm{Cl}, \mathrm{Kam})$, we show the planar projections of the $\Delta \chi^{2}=9$ theoretical three-dimensional regions for the BU, TL and DS models, respectively. Also shown are the projections of the $\Delta \chi^{2}=9$ experimental ellipsoids (dashed ellipses). A further projection onto the coordinate axes would yield the $3 \sigma$ theoretical and experimental errors.

The strong incompatibility between theory and experiment in the BP model is evident in Fig. 1. The theoretical errors are here larger than the experimental ones, but the distance between the two central values keeps the two $\Delta \chi^{2}=9$ ellipsoids in the (Ga, Cl, Kam) space widely separated. From Fig. 1 it is easy to convince oneself that to neglect the theoretical correlations would be a gross approximation. In this case the theory-experiment compatibility would get worse.

As can be seen in Fig. 2, the TL model is also in a very poor agreement with the experimental data (even if better than BP). It is clear that even stretching the theoretical uncertainties to their $3 \sigma$ limits, this model "misses" the bulk of the experimental allowed region. The mismatch is particularly evident in the $(\mathrm{Cl}$, Kam) plane.

In Fig. 3 we finally consider the DS model, characterized by relatively low neutrino rates. Here the correlation effects appear particularly important in the (Ga, Kam) and (Cl, Kam) planes: by neglecting them, one would obtain an artificially large superposition between the theoretical and experimental confidence regions. Again we see that in the $(\mathrm{Cl}, \mathrm{Kam})$ plane the ellipse describing the DS model does not point towards the data. Since the systematic errors of the Homestake experiment are subject to an endless debate, it is important to note that in the DS model we find only a marginal agreement with the data, even in the (Ga, Kam) plane.

The results reported in Table 8 and the discussion of Figs. 1-3 demonstrate that the present experimental neutrino rates cannot be convincingly reconciled with the standard solar model predictions, even stretching the theoretical uncertainties of low-rate models to their $3 \sigma$ limits. The correlation of the theoretical errors has proved to be crucial in this respect. 


\section{The "central temperature" parametrization}

As we have seen in the previous section, the theoretically allowed domain in the rate space could be substantially enlarged if the correlations were - uncorrectly - ignored. Conversely, the popular parametrization of the neutrino flux uncertainties in terms of the central temperature $T_{C}$ of the Sun (see, e.g., Refs. [16, 17]) has the effect of reducing the theoretical domain and overestimating the correlations.

In its extreme form, this parametrization relates all possible neutrino flux variations to the single "variable" $T_{C}$ through a power law dependence

$$
\phi_{i}(t)=\phi_{i} t^{\beta_{i}} \quad\left(t=1+\Delta T_{C} / T_{C}\right),
$$

where the temperature exponents $\beta_{i}$ can be estimated through a Monte Carlo procedure, as in Refs. $[16,12]$. We point out that in a linear approximation the values of $\beta_{i}$ are not independent, being constrained by the analogue of Eq. (2) at constant luminosity

$$
\frac{\sum_{i=1}^{8}\left(Q-2 \bar{E}_{i}\right) \phi_{i} \beta_{i}}{\sum_{i=1}^{8}\left(Q-2 \bar{E}_{i}\right) \phi_{i}}=0
$$

A possible set is $\left\{\beta_{i}\right\}_{i=1, \ldots, 8}=\{-1.1,3,5,8,18,27,32,32\}$, which satisfies the above equation at the $4 \%$ level using $\mathrm{BU}$ or $\mathrm{BP}$ fluxes.

If large (a few per cent) $T_{C}$ variations are allowed, then the linear expansion $\phi_{i} \simeq \phi_{i}\left(1+\beta_{i} \Delta T_{C} / T_{C}\right)$ is not adequate and the full power law must be used. To guarantee the validity of the energy production equation (1) at any temperature, we prefer to rescale all fluxes with a normalization factor $\mathcal{N}(t)$

$$
\phi_{i}(t)=\mathcal{N}(t) \phi_{i} t^{\beta_{i}} \quad, \quad \mathcal{N}(t)=\frac{\sum_{i=1}^{8}\left(Q-2 \bar{E}_{i}\right) \phi_{i}}{\sum_{i=1}^{8}\left(Q-2 \bar{E}_{i}\right) \phi_{i} t^{\beta_{i}}}
$$

Notice that, due to Eq. (10), $\mathcal{N}(t)-1$ is second order in $\Delta T_{C} / T_{C}$.

Now we discuss pros and cons of the $T_{C}$ parametrization. At first order, it implies that the matrix $\alpha_{i k}$ is a tensor product of two vectors

$$
\alpha_{i k}=\frac{\partial \ln \phi_{i}}{\partial \ln X_{k}}=\beta_{i}\left(\frac{\partial \ln T_{C}}{\partial \ln X_{k}}\right)
$$


However, it turns out that the above factorization can be approximately obtained only for submatrices of $\left\{\alpha_{i k}\right\}$. This simply means that $T_{C}$ variations cannot simulate the effects of all astrophysical uncertainties at the same time ${ }^{4}$, and at least a mixed $\left(T_{C}+\right.$ some $\left.X_{k}\right)$ approach is required [18] [12]. In particular, the errors induced by nuclear cross-section factors like $S_{17}$ and $S_{34}$ have sizeable components "orthogonal" to the $T_{C}$ uncertainties.

We now continue the discussion with the help of Fig. 4. In this figure we show, in the same planes as in Figs. 1-3, the one-dimensional theoretical domains allowed by $T_{C}$ variations ( $\Delta T_{C}$ lines), together with the central values of three standard (BP, TL, DS) and some non-standard solar model predictions. The $T_{C^{-}}$ dependent rates were calculated with the help of Eq. (11). The (almost straight) $\Delta T_{C}$ lines are conventionally normalized to give $\Delta T_{C}=0$ at the $\mathrm{BP}$ model, and have approximately the same slope as the main axes of the slanted ellipses of Figs. 1-3.

Four representative neutrino rate predictions involving non-standard (i.e. unjustified) assumptions are shown in Fig. 4. They correspond to models with artificially high-helium (High- $Y$ ) or low-metal (Low- $Z$ ) primordial abundance, zeroed $S_{34}$-factor $\left(S_{34}=0\right)$ or B-flux $\left(\mathrm{No}^{8} \mathrm{~B}\right)$, as considered in Ref. [5]. The first three such models lie approximately along the $\Delta T_{C}$ lines (like BP, TL and DS), and this evidences the usefulness of the $T_{C}$-parametrization in charting both standard and non-standard solar models. The fourth one $\left(\mathrm{No}^{8} \mathrm{~B}\right)$ is however considerably far from the $\Delta T_{C}$ lines, showing that changes in the nuclear reaction network can induce shifts orthogonal to $\Delta T_{C}$. This indicates the limits of the simple $T_{C}$ parametrization: too strong correlations, too narrow theoretical allowed regions. It would be better to think of the $\Delta T_{C}$ lines as of bands, with as large a $(3 \sigma)$ width as needed to cover the BP, TC and DS ellipses in Figs. 1-3.

Such bands are limited by solid slanted lines in Fig. 5. They represent the envelopes of the ellipses due to the $T_{C}$-dependent theoretical errors, obtained by applying our analytical prescriptions to the fluxes of Eq. (11) at any $T_{C}$. Concerning $\Delta \ln X_{k}$, we used the most conservative estimates. In the (Ga, Cl, Kam) space, the resulting theoretical allowed region is cone-like, and intersects the experimental ellipsoid only marginally. Its closest approach is at $\Delta T_{C}=-6.5 \%$, with $\chi^{2}=14.7$, still a very high value. Thus, to fictitiously introduce a new degree of freedom $\left(T_{C}\right)$ is of no real help. Note that, even allowing for both low $T_{C}$ and a larger experimental $\mathrm{Cl}$ rate, one can improve the fit in the $(\mathrm{Cl}, \mathrm{Kam})$ plane only at the price of getting a worse fit in the $(\mathrm{Ga}, \mathrm{Cl})$ plane. The rôle of the gallium data in excluding astrophysical solutions of the solar neutrino problem will be hopefully strengthened by the scheduled (summer 1994) GALLEX calibration by means of a radioactive source.

\footnotetext{
${ }^{4}$ Obviously, detector cross section uncertainties are a priori independent of $T_{C}$.
} 


\section{Conclusions}

In this paper we have faced the problem of an analytical calculation in matrix form of the theoretical errors affecting the solar neutrino fluxes. After an explicit description of the approach, we have thoroughly compared our output with the numerical results of Monte Carlo simulations. It is evident that the approach can be easily extended to cover new neutrino experiments and/or the uncertainties induced by a more refined parametrization.

We have shown that the correlations among theoretical errors play an important rôle in determining the statistical significance of the solar neutrino deficit. It is misleading to neglect the off-diagonal errors, the relevance of these correlation effects being more significant when lower theoretical rates are provided by the solar model. We have studied the predictions of three independent standard solar models, and charted standard and non-standard solar models with the help of the $T_{C}$-parametrization. Even in the most favourable cases, the theory-experiment agreement is only marginal.

Theoretical solutions to this discrepancy (if one wants to avoid new neutrino physics) are forced to involve also effects orthogonal to $\Delta T_{C}$. However, we do not know effects able to shift considerably the theoretical predictions towards the

experimental data. Moreover, even unexpectedly large systematic errors in the chlorine experiment cannot be considered as decisive as could be naïvely thought.

Finally, let us underline how clearly our representation in the space ( $\mathrm{Ga}, \mathrm{Cl}$, Kam) shows all these features, and of what considerable help it is in understanding the interplay between possible shifts of the theoretical and of the experimental allowed regions.

\section{Acknowledgements}

One of us (E.L.) wishes to thank the CERN Theory Division for kind hospitality during all stages of this work.

\section{Note added in proof}

While completing this work, we become aware of a recent paper by E. Gates, L.M. Krauss and M. White [19], which also addresses the problem of analytically estimating the correlation matrices, in particular to get improved MSW fits. In the present paper we have instead focused our interest on standard and nonstandard solar models, having already applied the full error matrix estimate in our MSW analyses [9, 10, 11]. 


\section{References}

[1] GALLEX Collaboration, P. Anselmann et al., Phys. Lett. B327 (1994) 377.

[2] SAGE Collaboration, J.N. Abdurashitov et al., Phys. Lett. B328 (1994) 234.

[3] R. Davis, talk given at the XVI International Conference on Neutrino Physics and Astrophysics - Neutrino '94, Eilat, Israel, 1994, to appear in the proceedings.

[4] Kamiokande Collaboration, as presented by Y. Suzuki at the same Conference as Ref. [3].

[5] J.N. Bahcall and R. Ulrich, Rev. Mod. Phys. 60 (1988) 297.

[6] J.N. Bahcall and M.H. Pinsonneault, Rev. Mod. Phys. 64 (1992) 885.

[7] S. Turck-Chièze and I. Lopes, Astrophys. J. 408 (1993) 347; see also: S. Turck-Chièze, W. Däppen, E. Fossat, J. Provost, E. Schatzman and D. Vignaud, Phys. Rep. 230 (1993) 57.

[8] A. Dar and G. Shaviv, Technion preprint PH-94-5 (1994), submitted to Phys. Rev. Lett.

[9] G.L. Fogli and E. Lisi, Astropart. Phys. 2 (1994) 91.

[10] G.L. Fogli, E. Lisi and D. Montanino, Phys. Rev. D49 (1994) 3626.

[11] E. Lisi, in the Proceedings of the International Topical Workshop: Solar Neutrino Problem - Astrophysics or oscillations? (Lab. Naz. del Gran Sasso, L'Aquila, Italy, 1994), LNGS publication, ed. by V. Berezinsky and E. Fiorini.

[12] V. Castellani, S. Degl'Innocenti, G. Fiorentini, M. Lissia and B. Ricci, Univ. of Ferrara preprint INFNFE-3-94 (1994), submitted to Phys. Rev. D.

[13] A. Kovetz and G. Shaviv, Astrophys. J. 426 (1994) 787.

[14] V. Castellani, S. Degl'Innocenti, G. Fiorentini, M. Lissia and B. Ricci, Phys. Lett. B324 (1994) 425.

[15] D.R.O. Morrison, Int. J. Mod. Phys. D1 (1992) 281.

[16] J.N. Bahcall, Neutrino Astrophysics (Cambridge University Press, Cambridge, 1989). 
[17] S.A. Bludman, D.C. Kennedy and P.G. Langacker, Phys. Rev. D47 (1993) 2221.

[18] N. Hata and P. Langacker, Phys. Rev. D48 (1993) 2937; Phys. Rev. D49 (1994) 3622; Univ. of Pennsylvania preprint UPR-0592T (1993).

[19] E. Gates, L.M. Krauss and M. White, Univ. of Chicago preprint FermilabPub-94/176-A (1994).

\section{Figure captions}

Fig. 1: Projections of the $\Delta \chi^{2}=9$ experimental and theoretical confidence ellipsoids onto the neutrino rate planes ( $\mathrm{Ga}, \mathrm{Cl}),(\mathrm{Ga}, \mathrm{Kam})$ and $(\mathrm{Cl}, \mathrm{Kam})$. The theoretical estimate refers to the Bahcall and Pinsonneault model (BP).

Fig. 2: As in Fig. 1, but for the solar model of Turck-Chièze and Lopes (TL).

Fig. 3: As in Fig. 1, but for the solar model of Dar and Shaviv (DS).

Fig. 4: $T_{C}$ parametrization: one-dimensional domains allowed in the $(\mathrm{Ga}, \mathrm{Cl})$, ( $\mathrm{Ga}, \mathrm{Kam})$ and $(\mathrm{Cl}, \mathrm{Kam})$ planes by $-10 \%<\Delta T_{C}<+3 \%$ (oblique lines). Also shown are the rates (central value only) predicted by three standard (BP, TL, DS) and four non-standard (High- $Y$, Low- $Z, S_{34}=0, N_{0}{ }^{8} \mathrm{~B}$ ) solar models. See text for details.

Fig. 5: Total theoretical $3 \sigma$ range (solid lines), allowed by all known solar model uncertainties at any $T_{C}$. Also shown are the projections of the $\Delta \chi^{2}=9$ experimental confidence ellipsoid. 


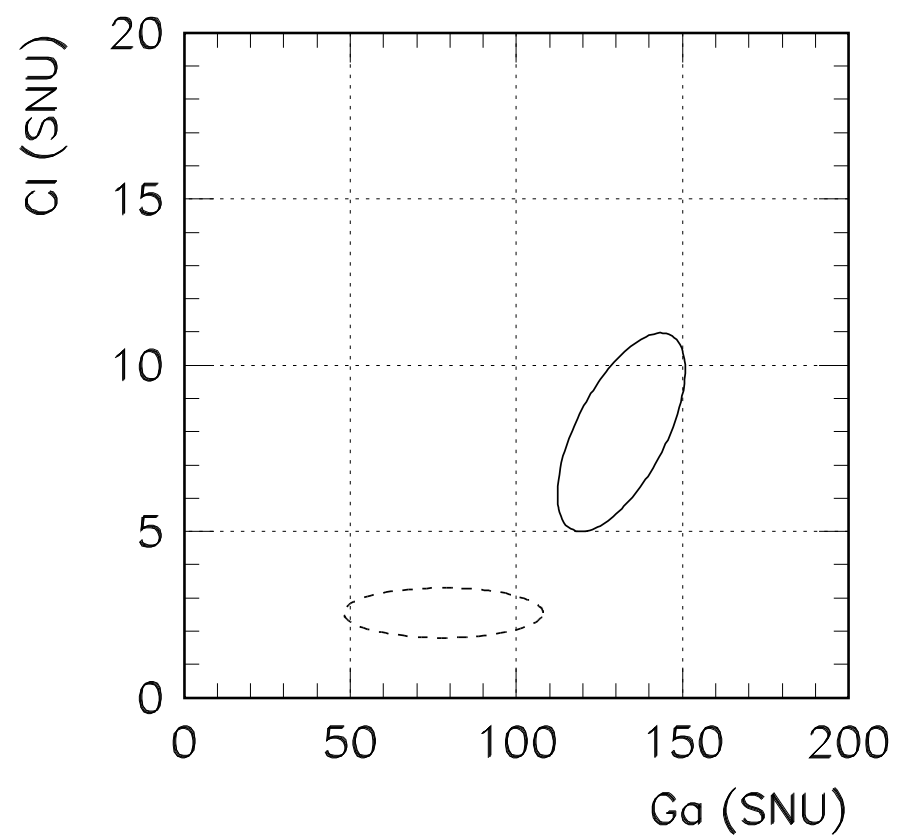

BP model

exp. data
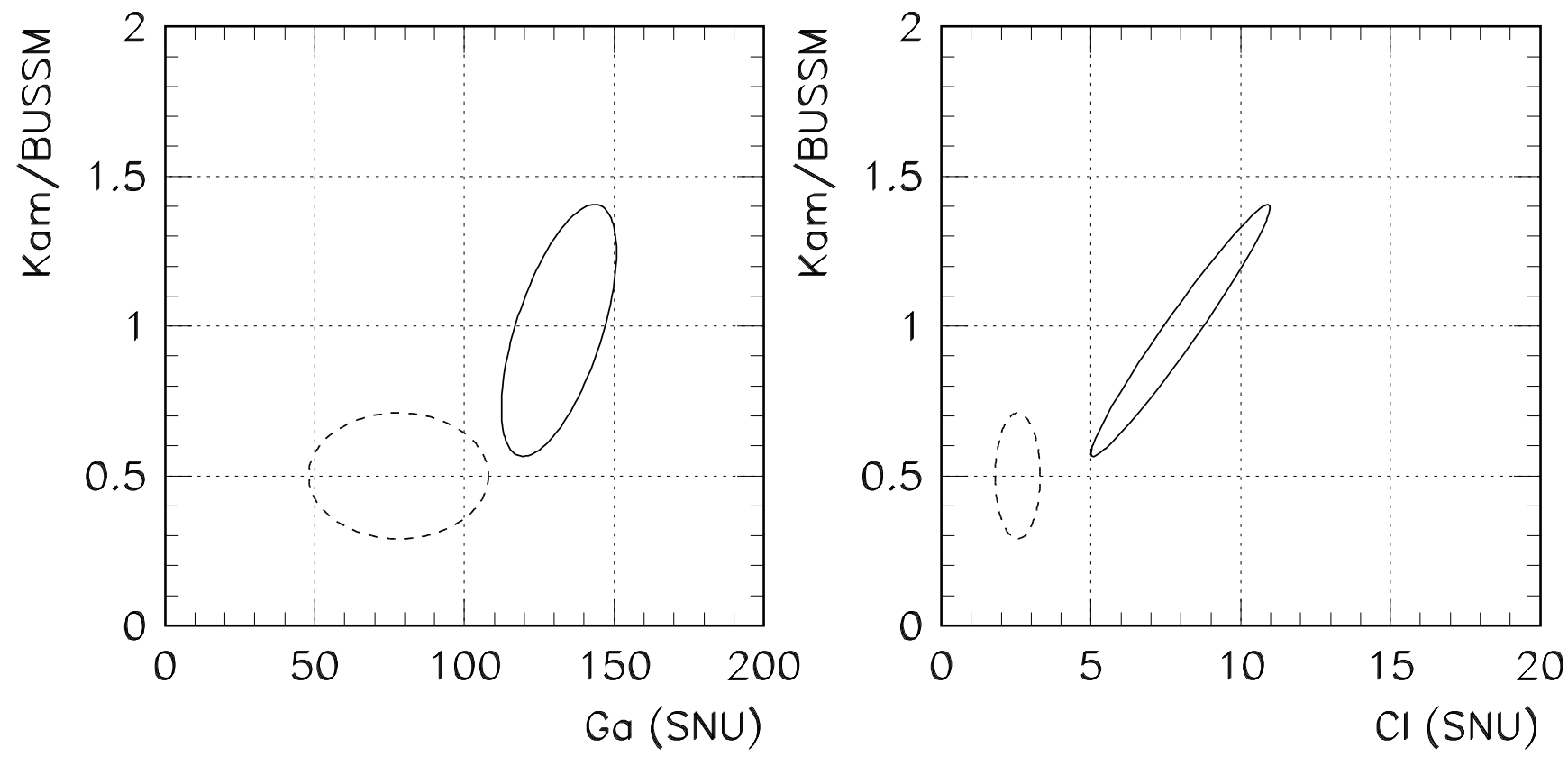

Fig. 1 


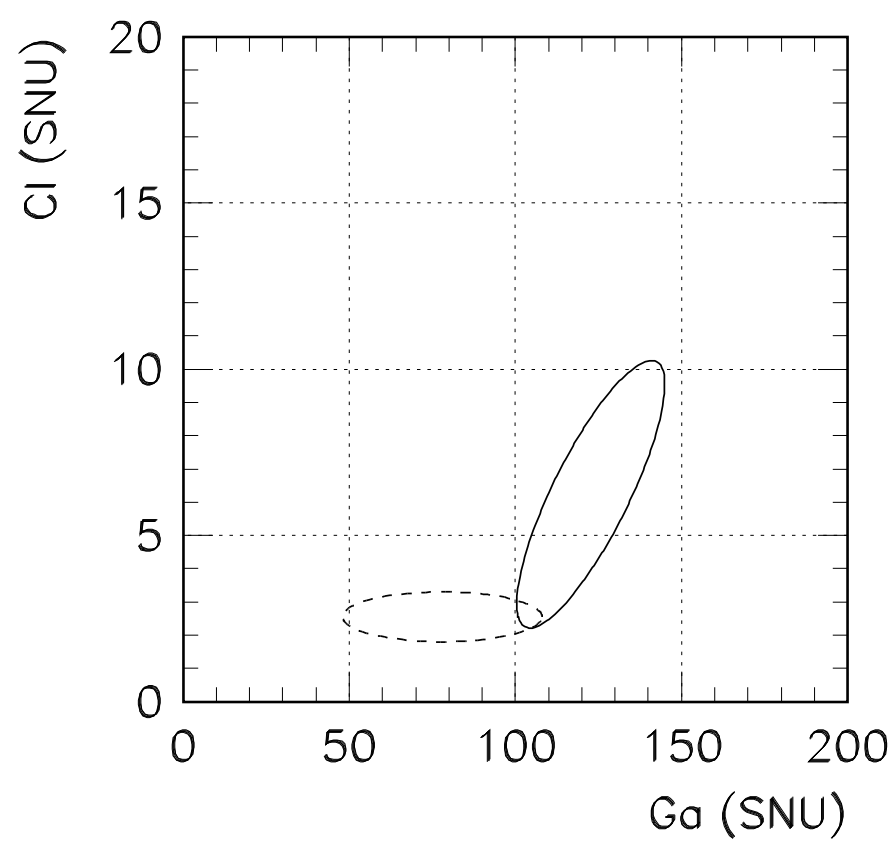

TL model

exp. data
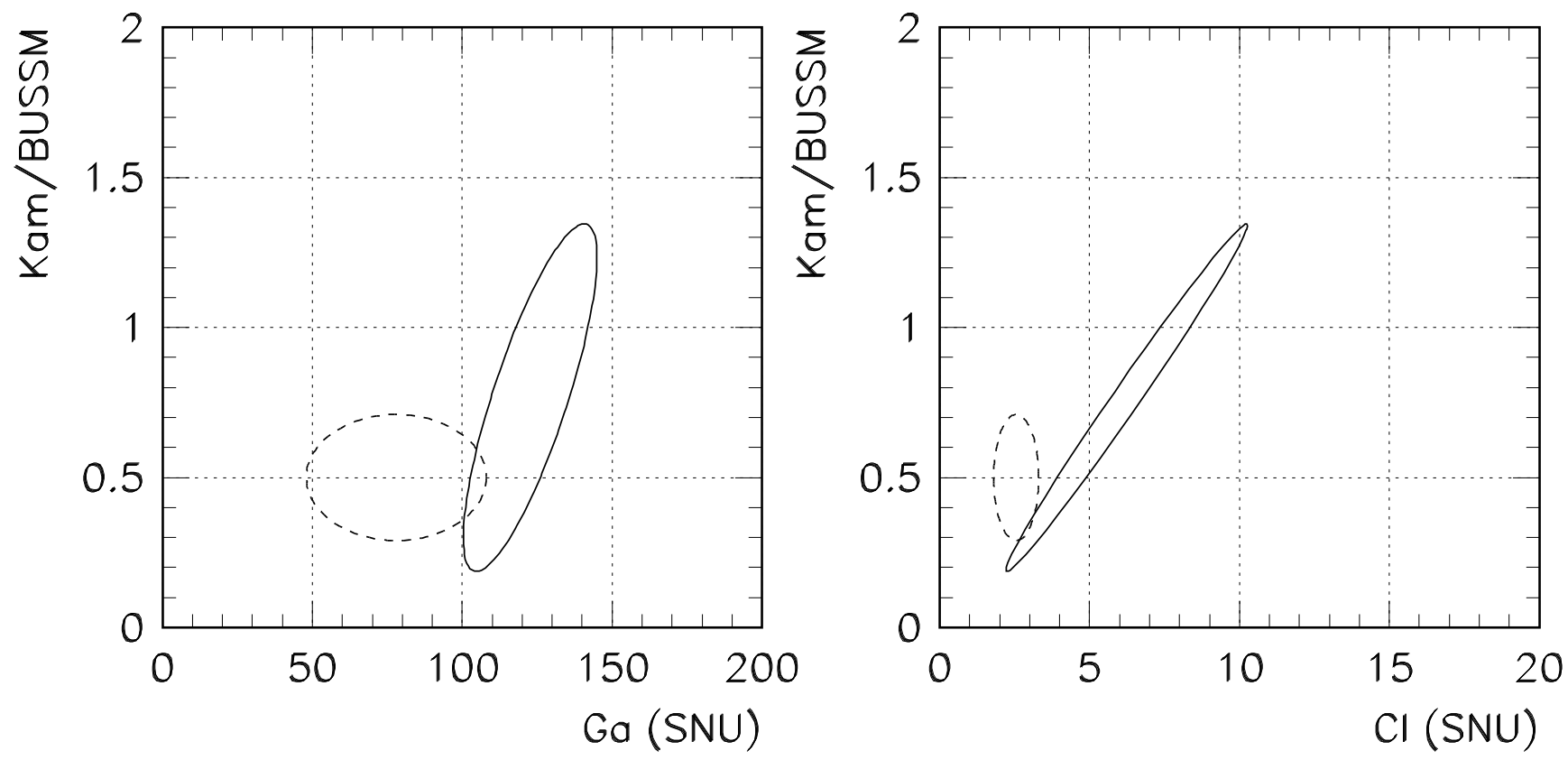

Fig. 2 


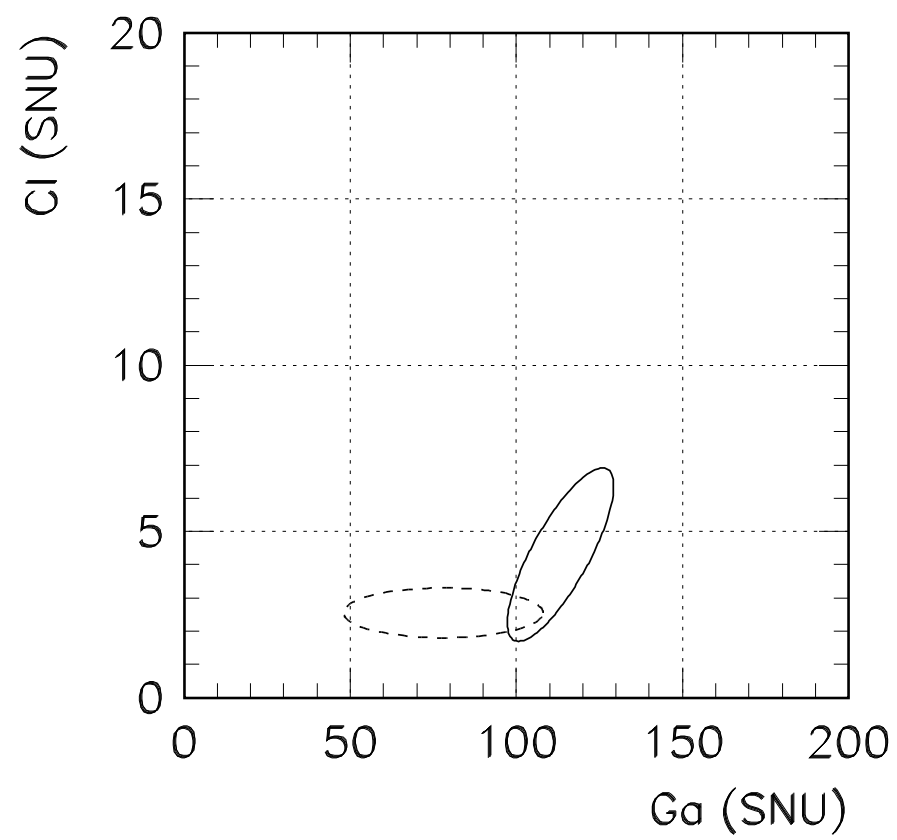

DS model

exp. data
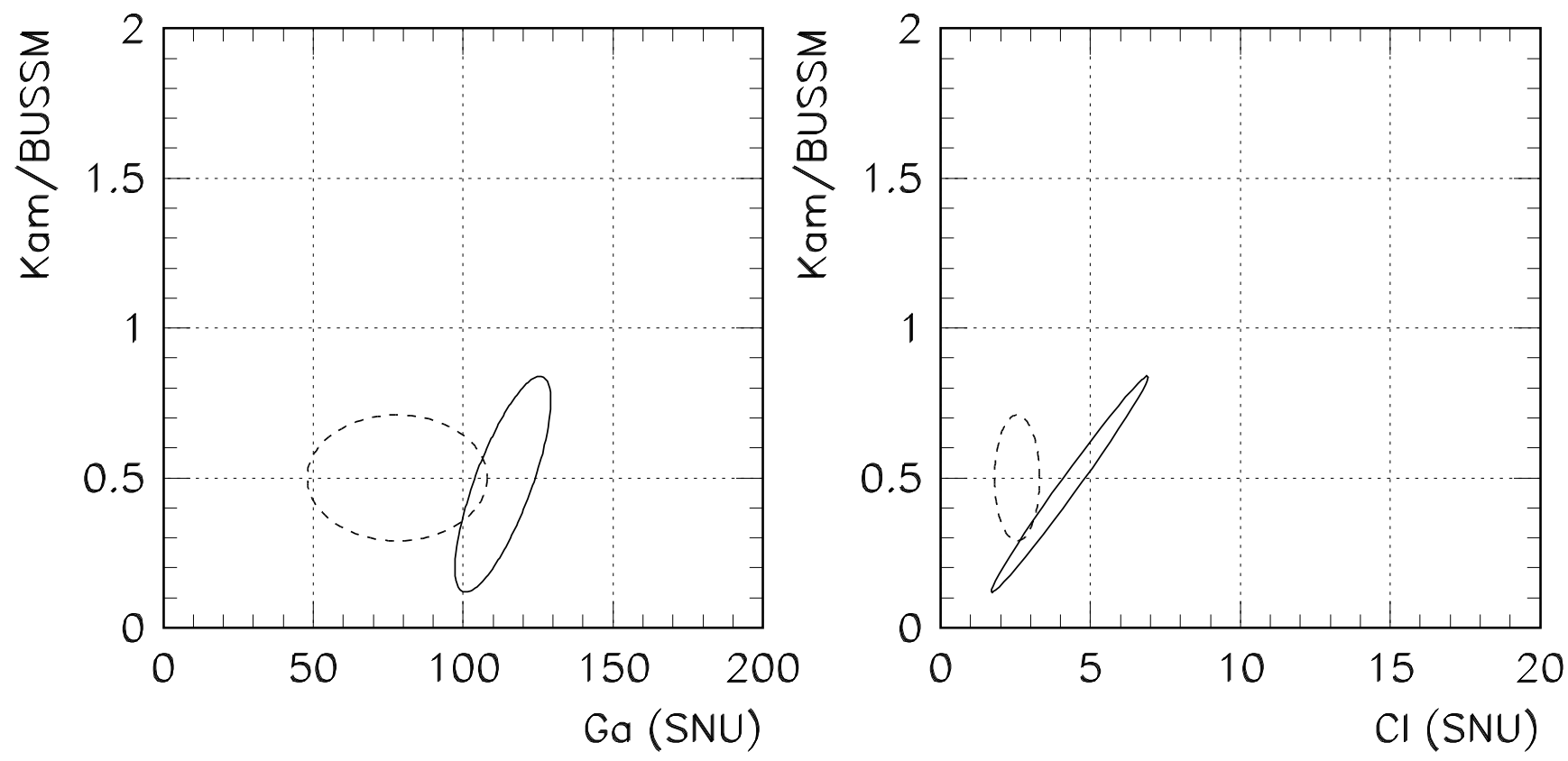

Fig. 3 


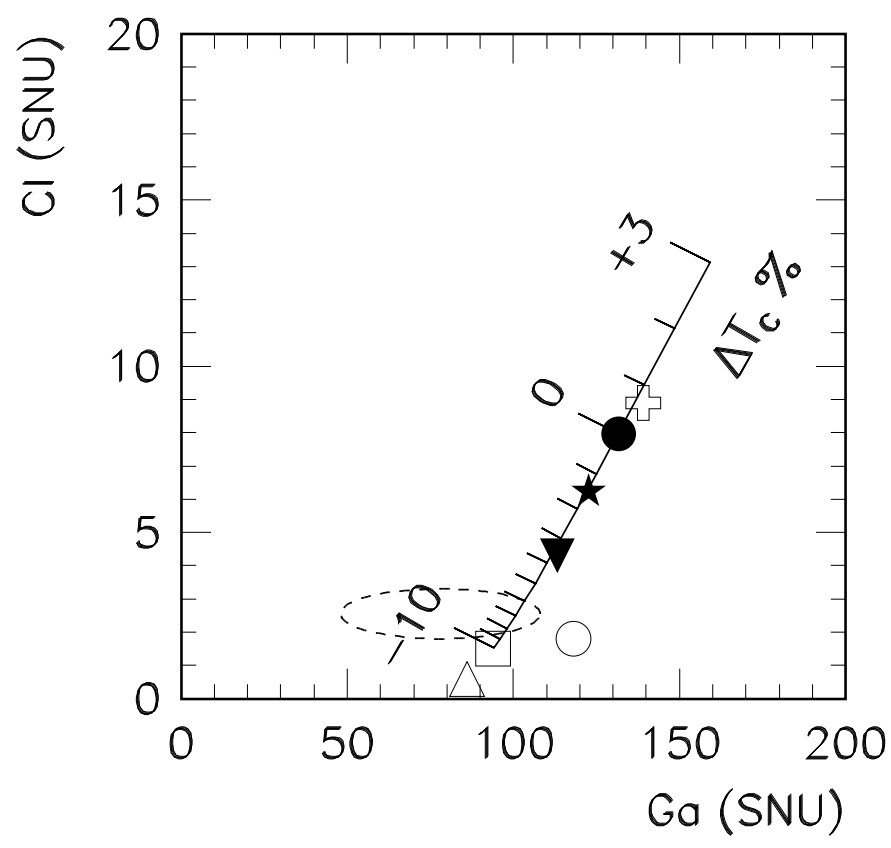

* BL Standard

$\nabla$ DS Models

\section{High $Y$}

Low Z Non-standard $\mathrm{S}_{34}=0 \quad$ Models

No ${ }^{8} \mathrm{~B}$

\section{Exp. data}
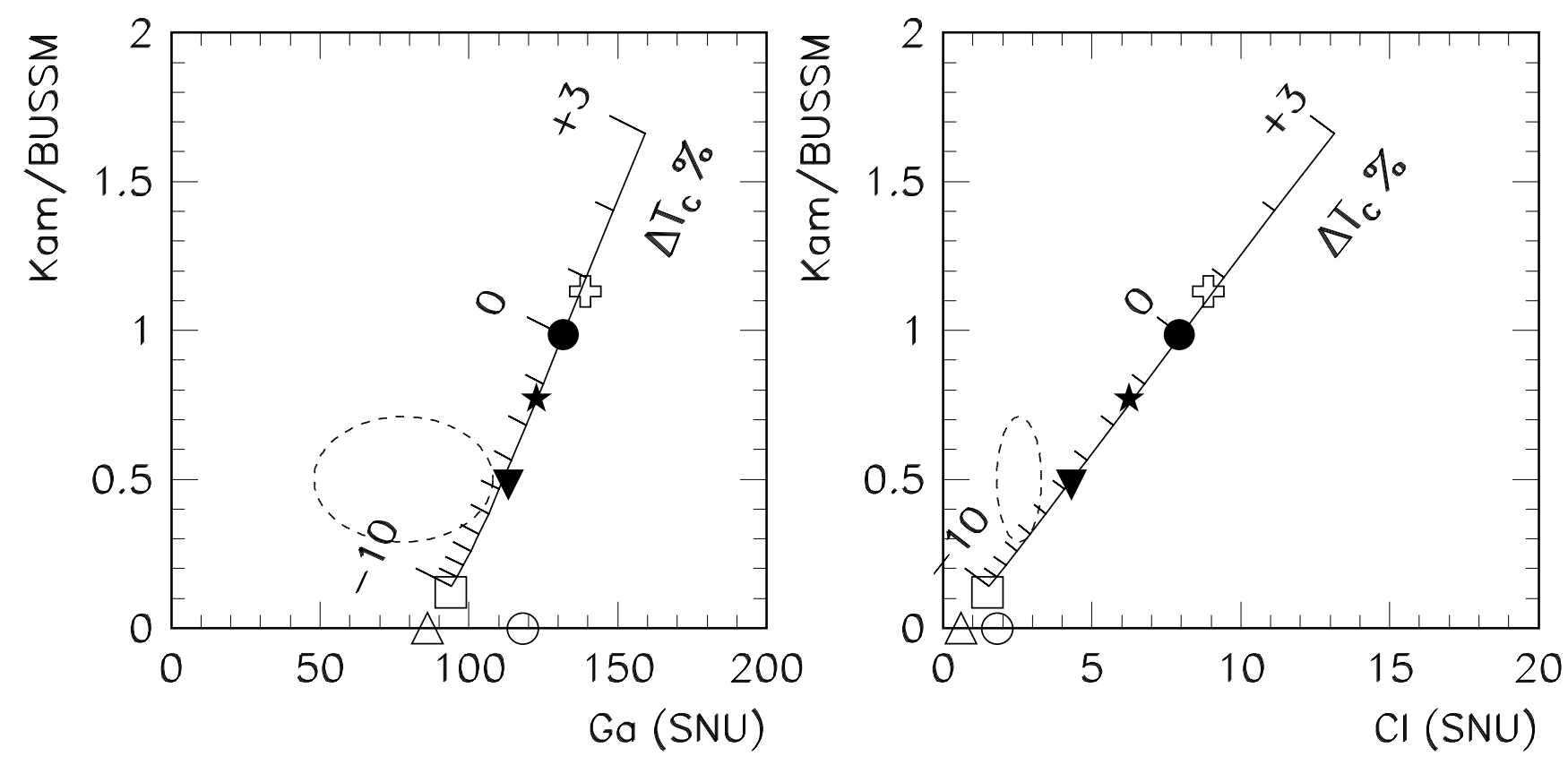

Fig.4 


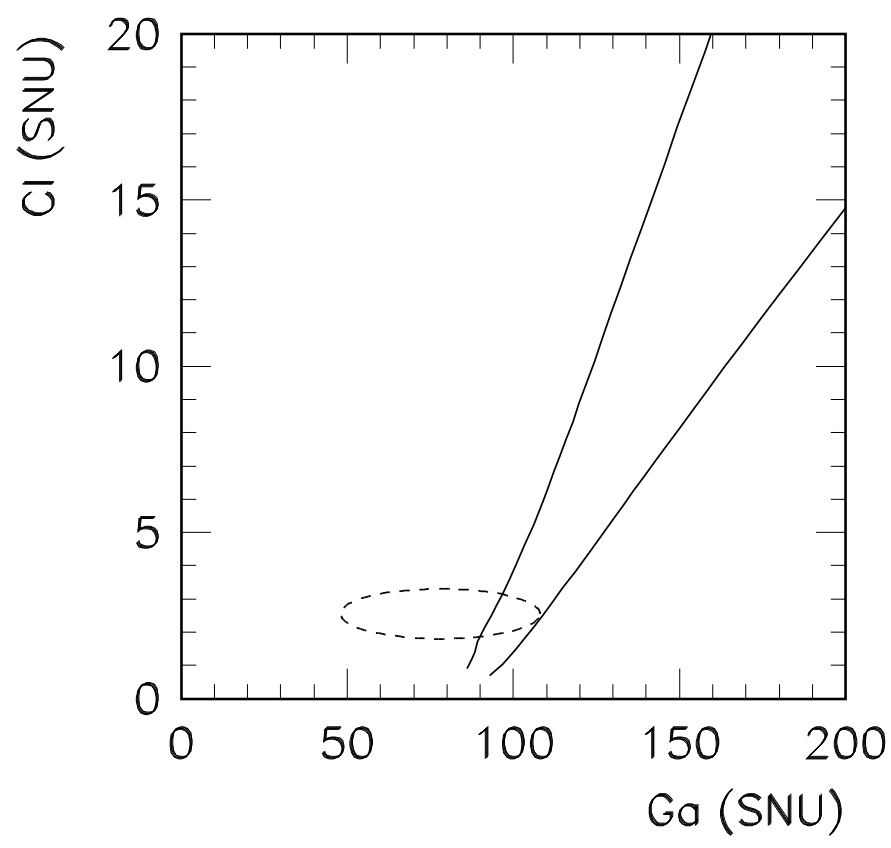

Th. range

Exp. data
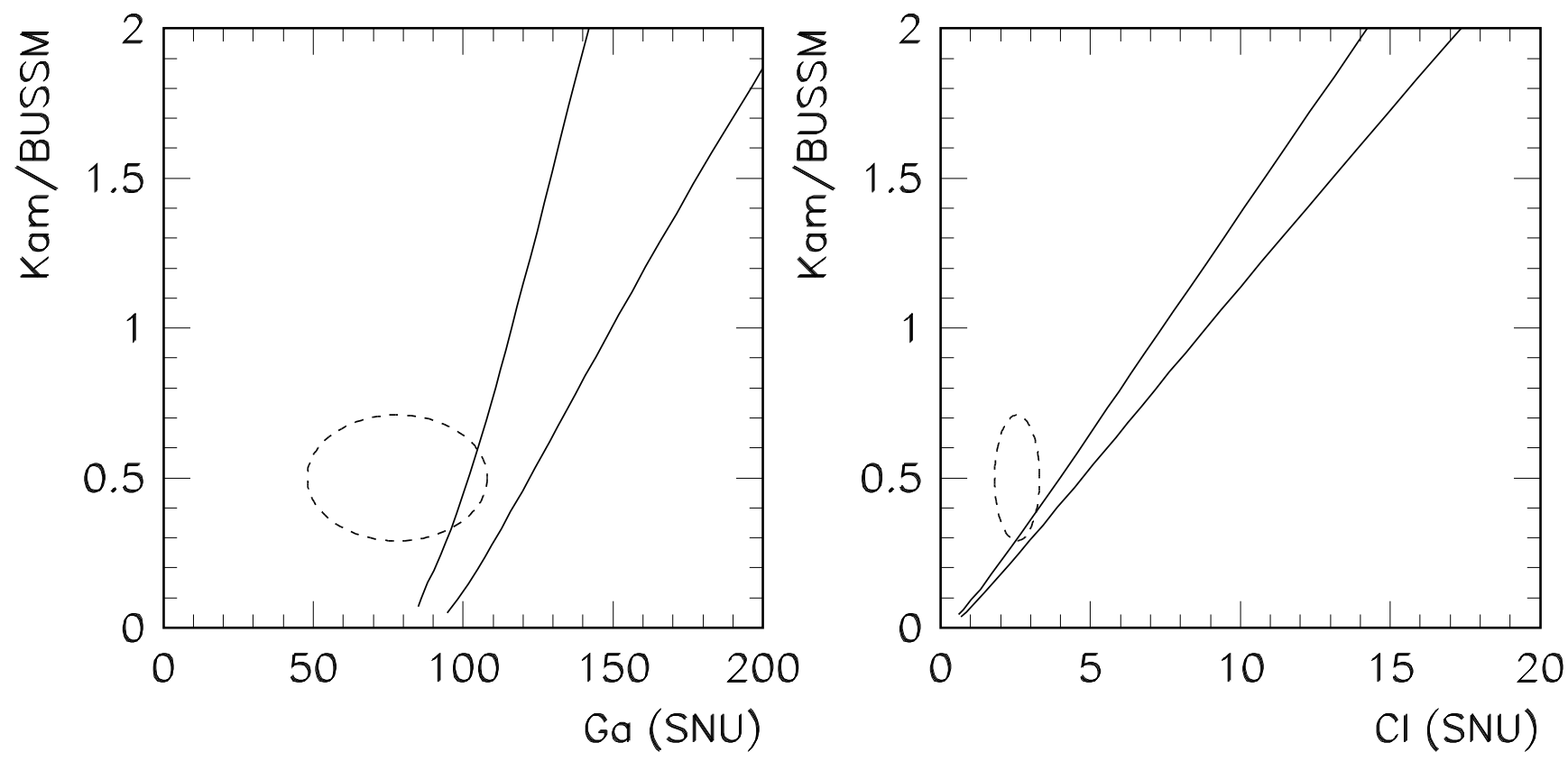

Fig. 5 